\title{
Telepsychiatry and Medical Students: a Promising Mental Health Treatment for Medical Student Use Both Personally and Professionally
}

\author{
Jennice A. Lavergne ${ }^{1} \cdot$ Matthew L. Kennedy ${ }^{2}$ \\ Accepted: 5 March 2021 / Published online: 13 April 2021 \\ (C) Springer Science+Business Media, LLC, part of Springer Nature 2021
}

\begin{abstract}
Purpose of Review We review recent findings on the use of telepsychiatry while investigating medical students' perceptions and willingness to use it for their mental health needs. We explore the impact of COVID-19 on medical school curriculums. We also investigate current education in medical schools surrounding telemedicine.

Recent Findings Medical students experience symptoms of anxiety at a greater rate than the general population. Major barriers to help-seeking behaviors are the refusal to seek treatment due to fear of incurring negative views from supervising faculty, time constraints, and the cost of counseling services. Those who do use telemental health resources have positive views and believe that it is an effective tool.

Summary Medical students are willing to use telepsychiatry for their personal mental health needs and recognize its value as a treatment modality that will be useful for their future patients. The telemedicine field is evolving but many medical school curriculums do not include education on telemedicine.
\end{abstract}

Keywords Medical students $\cdot$ Mental health $\cdot$ Telemedicine $\cdot$ Telemental health $\cdot$ Telepsychiatry

\section{Introduction}

Medical students are faced with multiple stressors during their education. During the pre-clerkship years, they are encumbered with academics, the constant pressure to succeed, and possible competition among classmates. During the clerkship years, medical students are exposed to patients with complex health issues, patients with acute or chronic medical issues, and potential grief from mortality. An exploratory analysis from 2019 showed that medical students overall, as compared with members of the general population of similar age, experience significantly more stress and lower mental health quality of life:

This article is part of the Topical Collection on Complex MedicalPsychiatric Issues

Jennice A. Lavergne

jennice.lavergne@gmail.com

1 Universidad Iberoamericana School of Medicine, Avenida Francia 129, Santo Domingo, DN 10203, Dominican Republic

2 WMed Health Psychiatry, 1717 Shaffer St, Ste 010, Kalamazoo, MI 49048, USA mental health and substance-related problems among medical students ranges at varying times during their clerkship training from 17 to $58 \%$, between 19 and $44 \%$ of medical students experience symptoms of anxiety, about $27 \%$ experience symptoms of depression, and over $25 \%$ of medical students have considered suicide, with $11 \%$ having seriously contemplated suicide within the last year [1]. As medical student debt burdens rise along with the cost of education, the potential for this debt to significantly impact students' well-being, career preparation and career choices, and thus the quality of care that they can provide to their current and future patients, also grows [2]. An empirical report from 2017 showed that medical students feeling psychologically and/or emotionally unsupported at their university, which increased over the years of medical training, was a predictor of psychological distress and burnout (this risk was reduced in those who felt supported) [3]. A 2016 research report measured alcohol dependence in 4402 medical students using the Alcohol Use Disorders Identification Test screening tool and found alcohol abuse/dependence was significantly more common in those with more than US $\$ 100,000$ in educational debt $(p<0.01)$. The report also showed that $80 \%$ had burnout, alcohol abuse/dependence, or depressive symptoms at the time the survey was conducted [4]. 
Despite all the evidence that speaks to medical students needing services, mental health care is seldom sought by these individuals. Many medical students are wary of privacy breaches, stigma, and their career trajectory being negatively impacted. It has been hypothesized that stigma-related barriers to seeking help may contribute to distress and/or burnout during medical students' future practice as physicians [3].

Medicine is constantly evolving, and as technology becomes more ingrained in people's daily lives, there is an opportunity to intertwine the two in order to provide support and treatment for growing mental health concerns, especially in the context of the COVID-19 pandemic. Telepsychiatry is the implementation of digital devices, web-based interventions, and other communication technologies to provide psychiatric services remotely [5]. Telepsychiatry was designed to provide access to people in areas where care for their mental health needs was limited or inaccessible [5]. It was initially implemented in the USA in 1956 by doctors at the University of Nebraska. Their goal was to provide consultation services, gather data about patients, and train residents from both the Norfolk State Hospital and the Nebraska Psychiatric Institute. Dr. Cecil Wittson used a two-way television system which allowed communication between different medical centers in Nebraska [6]. Medical students are often not exposed to telepsychiatry and the ways that it could help them provide care for patients in the future. As it is the second most commonly used form of telemedicine (aside from teleradiology), exposing students to telepsychiatry resources early in their medical training could prove to be useful [5].

In this article, we review the mental health burden of medical students, the impact of stigma on students' help-seeking behaviors, and explore how telepsychiatry may be implemented as part of medical school curricula.

\section{Mental Illness Stigma Among Medical Students}

The pursuit of a medical degree is a challenging journey to embark on. Rigorous courses, large amounts of debt, high volumes of work, and long study sessions are only a few of the stressors that medical students deal with. The impact that this has on students' mental health is important to analyze and understand in order to better support medical students as they embark on their journey to become physicians. A recent metaanalysis comprising 69 studies and 40,438 medical students worldwide concluded that the global prevalence rate of anxiety among medical students is $33.8 \%$, which is much higher than the prevalence within the general population [7]. Another study found that the prevalence rate of depression within the medical student community was $27 \%$ [8].

As previously stated, the rate of suicidal ideation of medical students is over $25 \%$ [1]. Though these numbers are concerning, many students do not seek treatment for their symptoms for fear of appearing incompetent or consequences that could impact their future as aspiring physicians [8]. 50.3\% of medical students from six different schools held the belief that residency program directors would pass over their residency application if they were cognizant of the fact that the student had mental health or emotional health issues [9]. While in a separate study, $74 \%$ agreed that their supervisors such as residents and faculty would see them in a less favorable way if they thought that the student had a mental or emotional problem and the same amount believed that residency directors would not give their residency application a chance [10]. In the survey conducted in six different medical schools, $46.4 \%$ of medical students agreed that patients who knew that the student was being treated for a mental health issue would not want them as a doctor [9]. In a survey conducted at one medical school, $84 \%$ agreed to this statement [10], while $10.3 \%$ of medical students agreed that receiving treatment for mental illness is a sign of weakness [9]. In a separate study, $12 \%$ of medical students also agreed with that statement [10]. These stigmatizing beliefs are just a few of the barriers to medical students seeking support and treatment for their mental health concerns, leaving affected medical students to suffer in silence.

Mental health is an ever-growing concern both nationally and globally. 46.6 million adults deal with mental illness on a national scale as of 2017 [11]. Medical students and physicians are an integral part of the medical community and are often tasked with aiding people with mental health resources, support, and treatment. This may be tainted, however, by their personal views of the importance of their own mental health [10].

Many medical students are dealing with mental illness alone, rather than seeking out help due to personal or public stigma. They are at the first part of the lengthy training that awaits them and shaping better attitudes towards seeking help rather than struggling secretly or alone is imperative. Martin and colleagues conducted a randomized study of an educational intervention and concluded that medical students' attitudes towards mental illness and psychiatry are positively impacted through the interaction with senior physicians who disclose their personal experience with mental illness [10]. The ability for medical students to connect with physicians who have dealt with mental illness and are open about their experiences can help shift their perceptions on seeking help for their own mental or emotional problems. After medical students in this study heard these senior physicians' stories, they were given time to break into small groups and discuss their thoughts, then later provided resources in their community that provided care for mental illness. Ninety-one percent of medical students reported that after hearing about these physicians' experiences, they would seek care if they needed it [10]. Of these students, $63 \%$ agreed that 
they would hide the fact that they sought out mental health treatment [10], highlighting that the personal stigma surrounding it is still prevalent.

\section{Telepsychiatry for Medical Students}

As students progress through medical school, they will find that they have a lot less free time as classes become more rigorous and their responsibilities increase. A lack of time is another important barrier to students seeking out treatment for mental health issues [12-14]. Through the evolution of technology, many people can have constant interactions with others, without the need for face-to-face communication. Telepsychiatry and telemental health resources may ease the burden on medical students' having to carve out a significant amount of time in their already busy schedules to receive inperson treatment. In 2018, a Pew Research Center study found that about $91 \%$ of Americans from ages 18 to 29 have a smartphone $[15,16]$. Implementing telemental health resources at their fingertips could prove to be beneficial if the resources are indeed effective.

A recent cross-sectional study examined the usefulness of telepsychiatry and other telehealth resources for medical students and other health professional students. Medical students' represented the majority of respondents in this study, 24 out of the 36 respondents or $66.7 \%$ [14••]. $44.4 \%$ of students received a telepsychiatry consultation for psychiatric medication, $36.1 \%$ utilized remote counseling services, and $19.4 \%$ reported using both services [14*0]. Aside from time constraints being a barrier to accessing in-person counseling services, students in this study also cited distance and cost, $55.5 \%$ and $63.9 \%$ respectively, as reasons telemental health was a more viable option for them to seek care [14.•]. This is in accordance with other literature that reported similar barriers to accessing mental health services specifically the cost of them $[12,13]$. As the prevalence of anxiety, depression, burnout, and suicide in medical students' continues to climb, it is imperative to provide a cost-effective and accessible resource that students can access and get the help and treatment that they need. However, in 2019, the Association for University and College Counseling Center Directors reported that 247 college counseling centers of the 473 surveyed did not provide any telehealth resources [17]. About $52.2 \%$ of college counseling centers do not provide these resources which could be beneficial for medical students' with limited time and money or even students on rotations away from their medical school and the college campus that houses services for student mental health [14••].

$94.4 \%$ of the students in the study conducted by Nobleza and colleagues found telehealth to be convenient and time saving. One respondent used the remote service to avoid being seen in the on-campus counseling center highlighting the issue highlighted previously of stigma as a deterrent to utilizing inperson counseling services [14.•]. A large majority of students also stated that the telehealth visits improved how they felt, reduced their distress or symptoms, and felt natural even though the delivery of the psychiatric or counseling services was delivered through an electronic interface [14••].

Overall, this cross-sectional study highlighted the effectiveness of telehealth, the willingness of students to use it, and the positive perceptions toward the personal use of it. It is important to note that this study included a small sample of 36 student respondents and though the evidence shows how telepsychiatry and telemental health resources can be advantageous for medical students it is indeed limited. Most of the research involving telepsychiatry and medical students is from the viewpoint regarding how they can use it for future patients. There is a scarce amount of literature on how medical students can use the resource for their own mental health needs, though the prevalence of anxiety, depression, and suicide is high within the medical student community highlighting the need for different types of interventions.

\section{Telemedicine and Medical Education}

Telemedicine is the delivery of medicine through a remote technology-based interface, as technology continues to evolve, the way it can be used clinically does as well [18]. Medical students are in the first part of their training to be physicians and are undoubtedly the future of medicine. The clinical applications of telemedicine are not limited to one specialty and as students discover which specialty they desire to train in, it is beneficial for them to learn more about telemedicine and its applications. It seems however that medical students have little knowledge or experience with telemedicine.

In a study investigating medical students' knowledge and confidence regarding telemedicine, $93 \%$ of respondents reported having no previous experience with telemedicine while in a separate study $52 \%$ of students stated that they had no prior experience with it aside from teleconsultations and

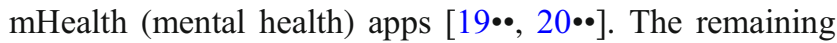
$7 \%$ had experiences with telemedicine including experience with a telestroke program and telemedicine while on rotations internationally $[19 \bullet \bullet]$. The students in this study responded to questions about their knowledge of telemedicine including efficacy, ethical concerns, telemedicine nomenclature, and appropriate body language during telemedicine visits, Medicare and Medicaid reimbursement, peripheral equipment, steps to take during a visit, and optimal office design for a telemedicine visit. Students received three educational modules about telemedicine over the course of 12 weeks, the most significant improvement in the knowledge scores before the modules to after was in office design, steps to take during a telemedicine visit, and peripheral equipment [19••]. Students recognize the 
importance of telemedicine; however, students were already halfway through medical school and the majority had no experience with it. Prior to training students, the lowest confidence score was regarding the statement "The quality of a telemedicine encounter is the same as an in-person visit." After training, the two categories that improved the most when comparing pre-training confidence scores to post-training were the students' confidence in conducting a patient encounter using telemedicine and the quality of a telemedicine encounter providing the same quality as an in-person visit [19••]. The educational modules used in this study were for an introductory telemedicine course, and the positive changes in the average confidence scores are important to note. Formal education about telemedicine seems to positively impact medical students' perceptions toward it and raise their confidence in their future use of it. A recent study found that $68 \%$ of medical students were interested in learning more about telemedicine and digital health while in medical school [20••]. The study also highlighted a variety of comments about the use of telemedicine, with the majority of their thoughts being in favor of learning more about the service, and integrating it into their already established classes rather than creating a new one strictly for telemedicine [20••].

Currently, about 80 medical schools in the USA currently include telemedicine as a part of their curriculum, which represents approximately 50 to $60 \%$ of medical schools nationally [21], while only $28 \%$ of physicians in the USA used telemedicine at all [21]. One of the key barriers to getting telemedicine in more schools is the lack of provider training for providers [21]. It is clear that there needs to be more training for medical students, residents, and senior physicians on telemedicine. The implementation of technology within medical education can potentially help medical students adapt to the changes that the medical community is facing and may continue to face due to COVID-19 as well as practice skills that can be helpful in managing telemedicine in the future [10].

\section{Impact of COVID-19 on Medical School Curriculum}

At the time of publication of this article, there are more than 35 million cases of COVID-19 and more than 1 million deaths worldwide (more than 7.6 million cases and more than 210,000 deaths in the USA alone). According to the Centers for Disease Control and Prevention (CDC), the virus that causes COVID-19 is thought to spread from person to person, mainly through respiratory droplets produced when an infected person coughs, sneezes, or talks. Spread is more likely when people are in close contact with one another (within about $6 \mathrm{ft}$ ) [22]. COVID-19 seems to be spreading easily and sustainably in the community and in many affected geographic areas [22]. In the absence of any pharmacological cure on the horizon, countries have resorted to the use of strict public health measures (lockdowns and social/physical distancing) to truncate the spread of further infection. The threat of COVID-19 has permeated the day-to-day lives of people all over the world, and medical students are no exception.

In most medical schools today, students convene regularly during the pre-clerkship years for interactive problem-solving or small group discussions and gain inpatient and outpatient experience as part of their curriculum during the clerkship years. Physical distancing is an extremely important public health measure [23] — consequently, this precludes students from gathering in learning studios, lecture halls, or small group rooms. In response to COVID-19, medical education faculty have quickly transitioned the entire pre-clerkship curriculum to online formats that include content in the basic sciences, health systems sciences, and even in behavioral sciences [24]. However, the loss of in-person collaborative experiences has the potential to become a detriment to education and many faculty have been emphasizing the irreplaceable value of attending class in-person, lauding the real-time feedback and back-and-forth that develop in class that are hard to replicate in online forums [25]. Primarily due to lack of personal protective equipment [PPE], medical students were sidelined in the early days of the pandemic, and in March of this year, the Association of American Medical Colleges issued guidelines that supported pausing clerkship rotations for medical students [26]. Today, even though many medical schools have allowed students to return, this has been at reduced capacity as many non-emergent surgical procedures and routine appointments have been canceled. Another issue is the cancelation of medical conferences where students have opportunities to present their research. While conference presentations are helpful ways to share knowledge with the medical community, they also assist in bolstering medical student resumes and applications for residency. With the loss of valuable experiences of clerkship rotations, collaborative experiences, and conference presentations - standards which helped previous generations become future doctors - the question arises of how students will evolve and integrate themselves into the medical community [25]. Students must be innovative in devising ways to exhibit their skills, work ethic, teamwork, and dedication to research, which only adds more pressure to the already burdensome process of medical training and securing a residency position.

\section{Conclusions}

Telepsychiatry is constantly evolving as new technology and research on the modalities implemented through various digital interfaces are being explored. Though the system has many ways in which it can improve with regard to users' concerns of privacy, confidentiality, and preference for an in-person visit, there are still a variety of ways where it can 
be advantageous. Telepsychiatry provides more opportunities for patients in need of help with their mental health concerns to receive care without the constant worry of traveling to a doctor's office, finding time in their schedules to fit in an appointment, or be concerned about cost.

The current global pandemic has impacted curricula at many medical schools, as well as brought up feelings of uncertainty in medical students regarding clinical rotations, standardized testing, and their futures as physicians. Luckily, with the uncertainty also comes the opportunity to learn more about how remote options through telemedicine can prove beneficial for their own well-being as well as that of their future patients. Providing students with more information about telemedicine can improve their willingness to use this resource. There is an opportunity to incorporate telemedicine in medical school curricula but also as an additional resource for student counseling services.

The research discussing mental illness in medical students, barriers to care, and help-seeking behaviors is plentiful; however, very few discuss how telepsychiatry and other telemental health resources could be used within this population to aid in providing tools, therapy to aid their mental and emotional health, and treat mental illness. Evidence reveals how telepsychiatry is positively perceived by medical students, as well as its convenience and cost-effectiveness; however, there is limited information on the subject which points to a gap in the literature and a need for more studies to be conducted on this topic. It would be beneficial for future studies to explore strategies to reduce self-stigma in medical students and the doctors who teach them, the impact of long-term use of telemental health resources, and telepsychiatry.

Acknowledgments The editors would like to thank Dr. Rachel Conrad for taking the time to review this manuscript.

\section{Declarations}

Human and Animal Rights Informed Consent This article does not contain any studies with human or animal subjects performed by any of the authors.

Conflict of Interest The authors declare no conflict of interest.

\section{References}

Papers of particular interest, published recently, have been highlighted as:

•- Of major importance

1. Fischbein R, Bonfine N. Pharmacy and medical students' mental health symptoms, experiences, attitudes and help-seeking behaviors. Am J Pharm Educ. 2019;83(10):2204-15. https://doi.org/10. 5688/ajpe7558.
2. Pisaniello MS, Asahina AT, Bacchi S, Wagner M, Perry SW, Wong ML, et al. Effect of medical student debt on mental health, academic performance and specialty choice: a systematic review. BMJ Open. 2019;9(7):1-15. https://doi.org/10.1136/bmjopen2019-029980.

3. McLuckie A, Matheson KM, Landers AL, Landine J, Novick J, Barrett $\mathrm{T}$, et al. The relationship between psychological distress and perception of emotional support in medical students and residents and implications for educational institutions. Acad Psychiatry. 2018;42(1):41-7. https://doi.org/10.1007/s40596-017-0800-7.

4. Jackson ER, Shanafelt TD, Hasan O, Satele DV, Dyrbye LN. Burnout and alcohol abuse/dependence among U.S. medical students. Acad Med. 2016;91(9):1251-6. https://doi.org/10.1097/ ACM.0000000000001138.

5. Chakrabarti S. Usefulness of telepsychiatry: a critical evaluation of videoconferencing-based approaches. World J Psychiatry. 2015;5(3):286-304. https://doi.org/10.5498/wjp.v5.i3.286.

6. Doarn CR. Telemedicine and psychiatry - a natural match. mHealth. 2018;4:60. https://doi.org/10.21037/mhealth.2018.12.04.

7. Quek TTC, Tam WWS, Tran BX, et al. The global prevalence of anxiety among medical students: a meta-analysis. Int J Environ Res Public Health. 2019;16(15). https://doi.org/10.3390/ ijerph16152735.

8. Mehta SS, Edwards ML. Suffering in silence: mental health stigma and physicians' licensing fears. Am J Psychiatry Resid J. 2018;13(11):2-4. https://doi.org/10.1176/appi.ajp-rj.2018.131101.

9. Dyrbye LN, Eacker A, Durning SJ, Brazeau C, Moutier C, Massie FS, et al. The impact of stigma and personal experiences on the helpseeking behaviors of medical students with burnout. Acad Med. 2015;90(7):961-9. https://doi.org/10.1097/ACM.0000000000000655.

10. Martin A, Chilton J, Gothelf D, Amsalem D. Physician selfdisclosure of lived experience improves mental health attitudes among medical students: a randomized study. J Med Educ Curric Dev. 2020;7:238212051988935. https://doi.org/10.1177/ 2382120519889352.

11. Mental Illness. https://www.nimh.nih.gov/health/statistics/mentalillness.shtml.

12. Rodriguez ML, Corse AK, Rosen LD. Mental health services use among medical students: perceived stigma and barriers to care. Med Sci Educ. 2017;27(2):267-72. https://doi.org/10.1007/s40670-0170392-6.

13. Moreira de Sousa J, Moreira CA, Telles-Correia D. Anxiety, depression and academic performance: a study amongst Portuguese medical students versus non-medical students. Acta Med Port. 2018;31(9):454-62. https://doi.org/10.20344/amp.9996.

14.• Nobleza D, Hagenbaugh J, Blue S, Stepchin A, Vergare M, Pohl CA. The use of telehealth by medical and other health professional students at a College Counseling Center. J College Stud Psychother. 2019;33(4):275-89. https://doi.org/10.1080/ 87568225.2018.1491362. This study examined the experience of medical students and other health professional students' experiences with telehealth resources. Four different areas were assessed including access, therapeutic alliance, satisfaction, and effectiveness regarding their experience.

15. Internet, social media use and device ownership in U.S. have plateaued after years of growth. https://www.pewresearch.org/ fact-tank/2018/09/28/internet-social-media-use-and-deviceownership-in-u-s-have-plateaued-after-years-of-growth/. Accessed 10 May 2020.

16. Lattie EG, Lipson SK, Eisenberg D. Technology and college student mental health: challenges and opportunities. Front Psychiatry. 2019;10(APR):1-5. https://doi.org/10.3389/fpsyt.2019.00246.

17. Reetz DR. The association for university and college counseling center directors annual survey. AUCCCD Annu Surv. Published online 2015:1-146. http://files.cmcglobal.com/AUCCCD 2013 Monograph_Public.pdf. 
18. Mechanic OJ, Persaud Y, Kimball AB. Telehealth systems. [Updated 2020 Sep 18]. In: StatPearls. Treasure Island (FL): StatPearls Publishing; 2020.

19.• Walker C, Echternacht H, Brophy PD. Model for medical student introductory telemedicine education. Telemed e-Health. 2019;25(8): 717-23. https://doi.org/10.1089/tmj.2018.0140. This article highlighted how formal education on telemedicine can improve medical students' confidence with the resource, willingness to use it, and overall knowledge of telemedicine. Explored lack of exposure medical students have to telemedicine.

20.• Edirippulige S, Gong S, Hathurusinghe M, et al. Medical students' perceptions and expectations regarding digital health education and training: a qualitative study. J Telemed Telecare. 2020. https://doi. org/10.1177/1357633X20932436. This study analyzed medical students' perceptions and expectations with the integration of digital resources such as telemedicine into their medical school curriculum and be used by them as future clinicians.

21. Theobald M, Brazelton T. STFM forms task force to develop a national telemedicine curriculum. Ann Fam Med. 2020;18(3): 285-6. https://doi.org/10.1370/afm.2549.
22. Coronavirus (COVID-19) frequently asked questions. https://www. cdc.gov/coronavirus/2019-ncov/faq.html\#Basics. Accessed 10 May 2020.

23. Social Distancing, Quarantine, and Isolation. https://www.cdc.gov/ coronavirus/2019-ncov/prevent-getting-sick/social-distancing. html. Accessed 10 May 2020.

24. Rose S. Medical student education in the time of COVID-19. JAMA - J Am Med Assoc. 2020;323(21):2131-2. https://doi.org/ 10.1001/jama.2020.5227.

25. Ferrel MN, Ryan JJ. The impact of COVID-19 on medical education. Cureus. 2020;12(3):10-3. https://doi.org/10.7759/cureus. 7492.

26. Important Guidance for Medical Students on Clinical Rotations During the Coronavirus (COVID-19) Outbreak. https://www. aamc.org/news-insights/press-releases/important-guidancemedical-students-clinical-rotations-during-coronavirus-covid-19outbreak. Accessed 10 May 2020.

Publisher's Note Springer Nature remains neutral with regard to jurisdictional claims in published maps and institutional affiliations. 